\begin{tabular}{l} 
International Journal of Social Research \\
(ISSN:2576-5531) \\
\hline
\end{tabular}

\title{
Multimodal Discourse Analysis of Coca-Cola's Print Advertising
}

\section{Mengyuan Bi}

Shanxi Normal University.

\begin{abstract}
With the development of the Internet, the expression of ${ }^{*}$ Correspondence to Author: information is not limited to words, but it develops into other Mengyuan $\mathrm{Bi}$ modes, such as images, sounds, actions, etc. This study is Shanxi Normal University. based on the visual grammar. The visual grammar proposed by Kress and Van Leeuwen is based on Halliday's systemic functional linguistics. Coca-Cola as a popular soft drink brand, its graphic advertising design can be said to be very mature and representative. This study is based on visual grammar How to cite this article:

to carry on the thorough analysis to the American Coca- Analysis of Coca-Cola's Print Advertising. Cola classic print advertisement, carries on the analysis to International Journal of Social Research, each print advertisement from the reproduction meaning, 2019;3:33

the interactive meaning and the composition meaning. The following conclusions can be drawn: 1. In the Coca-Cola print advertisement in the United States, the professional characteristics of the characters are obvious. 2. Advertising language can directly express the benefits of the product. 3 . The combination of image mode and language mode in the CocaCola print advertisement can be explained by visual grammar.

\section{eScîPub}

eSciPub LLC, Houston, TX USA. Website: https://escipub.com/
\end{abstract}

Keywords: Visual grammar; Coca-Cola; Print advertising 


\section{Background of Coca-Cola and Visual Gra- mmar}

\subsection{Background of Coca-Cola}

On 8 May 1886, Dr. John Pemberton from Atlanta, George, United States, stirred carbonated water with soda to make a dark syrup, and it had the effect of refreshing, sedation and reducing headache, the drink was Coca-Cola. At first, Coca-Cola was sold at a drugstore, with the first Coca-Cola selling an average of nine bottles a day for 5 cents.

Frank Robinson, an accountant at Coca-Cola, inspired naming from two ingredients in syrup. These two ingredients are the leaves of Coca and the fruit of Kola. In order to be uniform, Robinson changed the $\mathrm{K}$ of Kola to $\mathrm{C}$, then added a horizontal painting to the middle of the two words, and wrote the famous brand logo with Spence. In 1887, John Pemberton registered the Coca-Cola syrup and concentrate trademark at the U.S. Patent Office, acquired its intellectual property rights, and used coupons for the first time to promote Coca-Cola. But it's a bottling system that made the Coca-Cola showed up. The Coca-Cola Company sells the syrup to the bottling system, and then the syrup is mixed with the bottling system and then sold after bottling. The bottle-filling system is manufactured according to the requirements of Coca-Coca's company and Coca-Cola allows bottling systems to advertise using Coca-Cola's trademar. With this special bottle-filling system, the factory that produced Coca-Cola has flowered everywhere.

\subsection{Visual Grammar}

With the development of the Internet, the expression of information is not limited to words, but it develops into other modes, such as images, sounds, actions, etc. It is necessary to combine the unimodal study of language in dis- course linguistics with other fields such as vision and hearing $(\mathrm{Li}, 2003)$. The visual grammar proposed by Kress and Van Leeuwen is based on Halliday's systemic functional linguistics. Kress and Van Leeuwen think, the significance of multimodal research is that it clearly points out that the interest in the study of modes other than words and speech is inevitable rather than accidental, it is the center of the form of realistic communication, not just as a supplement to language research(Kress,2006). Halliday put forward three major functions of the language in the functional grammar: ideational function, interpersonal function, textual function. The "ideational function" refers to the function of the language to describe the "content" and the function of establishing the "logic". The "interpersonal function" refers to the function that language has the function of reflecting the relationship between the speaker and the hearer. The "textual function" refers to the function that language forms a text, and then it can realize the function of information transmission(Halliday, 1994).

Kress and Van Leeuwen extended the three meta functions of language proposed by Halliday to visual texts, thus constructing the theoretical framework of visual grammar and putting forward three meanings in visual grammar: representational meaning, interactive meaning, compositional meaning. These three meanings also construct an effective analytical framework for image text analysis.

\subsubsection{Representational Meaning}

According to whether the image has "vector", the reproduction meaning is divided into narrative representation and conceptual representation.

Kress and Van Leeuwen point out that describing the functions of the existing designing so- 
cial action expresses developing events, changing processes, or continuous spatial distribution. According to the different forms of the vector and the participants, the description and reproduction is subdivided into action process, reaction process, speech and mental process. The action process is considered to be one of the most basic forms in narrative reproduction. In this kind of narration, the actor and the action goal complete the narration of the action process together. In such studies, participants, as the most important part of the image, can be analyzed by location, form, size, etc. The reaction process is a description of the vector structure through the eyes or eyes, which is different from the action process, and the reaction process image is composed of the reactor and the phenomenon. The process of speech and psychology refers to the process of the narration of the relationship between the speaker and the sensor through the expression of speech and the reflection of the vector. This kind of narrative process is the most common in cartoons. The author often expresses the relationship between the speaker and the senser through the bubble of thinking and dialogue.

Different from narrative reproduction, conceptual reproduction expresses a more abstract narrative process. Kress and Van Leeuwen believe that conceptual reproduction has the function of designing social constructs, and subdivides it into: classificational process, analytical process and symbolic process. Generally speaking, the classificational process embodies the relationship between upper and lower levels or peers, the analytical process embodies the relationship between the whole and the part, and the symbolic process shows the implicit meaning behind the participants.

\subsubsection{Interactive Meaning}

As a medium for transmitting information, the image can establish the relationship between the image viewer and the image, and create interactive meaning, which affects the psychological state of the reader. In the realization of interactive meaning, contact, social distance, perspective, modality. These four aspects are the main analysis point.

In the contact analysis of the image, Kress and Van Leeuwen divide the image into demanding and giving, according to whether the participants in the image and the viewer of the image have eye contact or not, the producer of the image will influence the viewer by a definite eye, thus establishing the relationship between the viewer and the image participant. If the participant in the image has eye contact with the viewer from top to bottom, it means seeking obedience; If a participant in an image is in contact with the viewer in a manner that is from the bottom to the top, it means that sympathy or compassion is obtained. On the contrary, if an image does not determine its intention by looking, the viewer must receive meaning from other information provided in the picture, then the image is the type given. Social distance refers to the degree of familiarity between the elements in the image and the viewer. Social close viewers tend to regard participants as "friends", while social distance tends to make viewers think of them as "strangers" or "others". Kress and Van Leeuwen believe that different lenses can show different degrees of closeness. Generally speaking, if a close-up of an image presents a participant, then what is revealed is a closer relationship; on the contrary, if the frame of the image is large, the panoramic view and the vision of the participants are presented, then the social distance of the image is far away, and the social relations are not so close. 
In terms of perspective, different perspectives reflect the status difference between image viewers and image participants. The angle of level often gives the viewer a sense of presence and reflects the equal social relations; the perspective of top view shows that the viewer of the image is in a strong position; if it is a bottom view, the participant in the image is in a stronger position, and the viewer is in a weaker position. At the same time, the different perspectives also reflect the attitude towards the things in the image. The positive and intuitive image makes the viewer feel close and regards it as part of himself, while the side squint image allows the viewer to define it as "them" rather than "us".

In terms of modality, Kress and Van Leeuwen believe that modality is described by the de- gree of credibility of the image. Here, the image reflects the true degree of reality and its picture's color, detail, depth, lighting, and social factors

\subsubsection{Composition Meaning}

The composition meaning in visual grammar evolved from the "textual function" of the three meta functions of Halliday's language. It synthesizes the representational meaning and the interactive meaning, and emphasizes how the text embodies the whole thought through structural composition and cohesion, thus expressing the meaning that it wants to express. Spatial distribution, salience, framing and the use of color are few levels that can be used to describe the meaning of a composition.

\section{Analysis of Coca-Cola' s Print Adver- tisement}

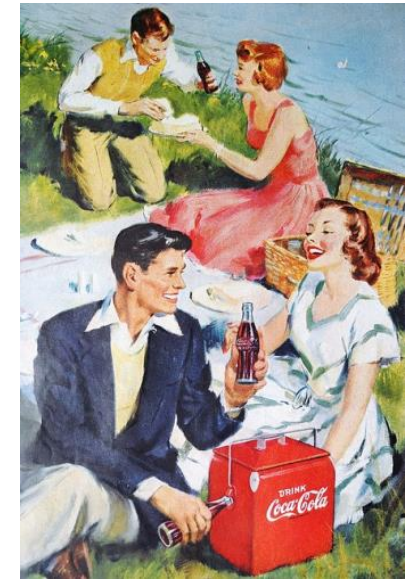

1

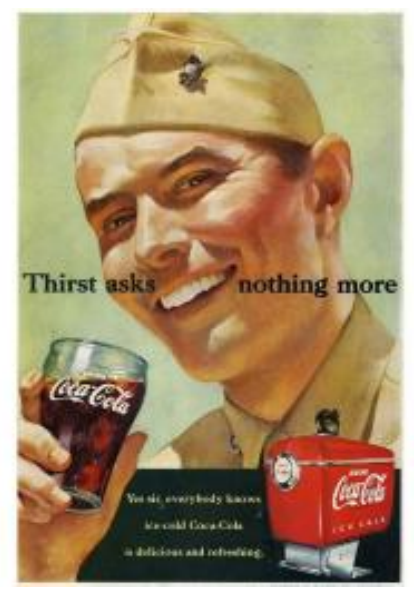

2

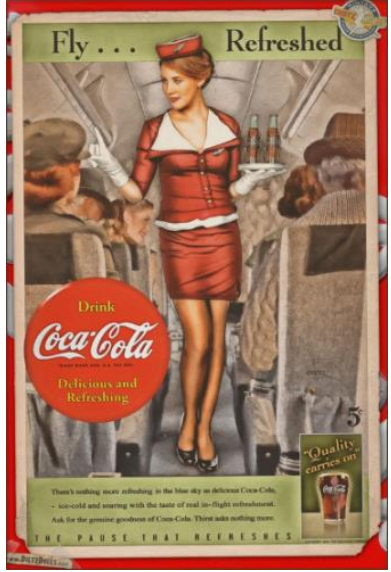

3

\subsection{The Analysis of Representation Meaning}

Figure 1 has an obvious vector, which belongs to narrative reproduction. In figure 1, there are four characters at the party. Among them, two close-up people meet in their eyes. The boy holds a bottle of Coca Cola in each hand, and the vector of holding coke in the left hand intersects the vector of the girl holding coke in the right hand, indicating that the boy is hand- ing the girl coke. The boy's right hand shows how coke is turned on. The two of them looked at each other and smiled at each other, indicating the joy of the party. In front of the picture is a Coca-Cola box with the slogan "Drink CocaCola". The advertising language is a direct indication of attitude. The boy behind the camera holds Coca Cola in his left hand, and the vector that produces tells us that the boy is 
pouring Coca Cola for the girl. The girl looked at the boy, and both of them were happy. This figure shows the happiness of a friend's party, with four of the vectors pointing to Coca-Cola, highlighting the focus of advertising.

Figure 2 has an obvious vector, which belongs to narrative reproduction. In figure 2 , the soldier looks at the audience and the vector points to the audience. In the front of the figure, one hand holds the Cola Cup to form a vector. There is an advertising language at the bottom of the picture: yes sir, every knows ice cold Coca-Cola is delicious and refreshing. Soldier has words on his mouths,: Thirst asks nothing more. Behind Asks is the soldier's mouth, which point to coke, and forms a vector with coke. It tells us to drink coke only if we are thirsty.

Figure 3 has an obvious vector, which belongs to narrative reproduction. As can be seen from the picture, the background is that on a traveling plane, the stewardess is providing drinks to the passengers. In Figure 3, the airline stewardess looked at the passenger, holding the plate to form a vector, on which there was Coke. In addition to the characters, there are other participants in the picture. At the bottom of the picture is the advertising language: "There's nothing more refreshing in the blue sky as delicious Coca-Cola. Ice-cold and soaring with the taste of real in-flight refreshment. Ask for the genuine goodness of Coca-Cola. Thirst asks nothing more." The advertising language not only expresses the characteristics of the Coke itself, but also the freshness of people after drinking the ice Coke, which proves that the Coke is not replaceable. On the left side of the picture is the brand logo of coke, and at the bottom right is the price of coke and the advertising language: quality carries on.

\subsection{The Analysis of Interactive Meaning}

The graphic advertisement in figure 1 adopts a flat-view shooting technique, giving the viewer a sense of equality. The distance between the character in the picture and the viewer belongs to the social close distance, which gives the viewer the feeling of being there. The four characters in the picture do not intersect with the viewer's line of sight, reflecting the attitude of "providing". Images provide viewers with some information, for example, a happy party between friends can't be separated from Coca-Cola; with Coca-Cola, everyone's party will be happier. The color saturation in Fig. 1 is high, showing the high situation, giving the viewer the real feeling.

The print advertisement of figure 2 adopts a head-up shooting method to provide an equal feeling to the viewer. The distance between the character in the figure and the viewer is personal close, giving the viewer a feeling of closeness. The soldier in the picture intersects the viewer's line of sight and expresses the attitude of asking. According to the analysis of the other participants in the print advertisement, we couId know that the information which soldier asked for was that they wanted viewers to drink Coca-Cola when they were thirsty. In figure 2 , the color of coke and the color of coke refrigerator are very bright, and the saturation of background and character color is also very high, which gives the viewer a feeling of reality. The plane advertisement of Fig. 3 adopts a head-up shooting method to provide an equal feeling to the viewer. The distance between the character in the figure and the viewer belongs to the close range of the society and gives the viewer an immersive feeling. The line of sight of the characters in the picture does not intersect with the viewer, reflecting the attitude of "provi- 
ding". The image should be provided with some information to the viewer. As can be seen from the figure, the information that the image wants to express is, the passenger wants to drink the coke in the flight of the figure, the airline stewardess 's dish is only coke, and it shows that the coke can't be replaced. In Fig. 3, the saturation of the color in brand logo of Coke, background and character are also very high, which gives the viewer a real feeling.

\subsection{The Analysis of Composition Meaning}

The male and female of the near-lens in Fig. 1 are in the center of the figure with high significance, and the composition shows the focus of the interactive meaning and the narrative meaning. Characters occupy one-half of the graph. The Coca Cola box in the front of the figure is perfectly combined with the central character through the vector, The color of coke box is bright red, which contrasts sharply with the background color, and the convex dominance is high.

The soldier in FIG.2 is in the center position and the significance is high. The composition shows the focus of the interactive meaning and the narrative meaning. Character occupies one-half of the graph. The soldier's words are in the center of the graph, between known and unknown information. Such expressions tend to arouse the curiosity of viewers. "yes sir, every knows ice cold Coca-Cola is delicious and refreshing." This sentence is located in the realistic position in the picture which reflects the reliability and authenticity of the discourse content. In figure 2, the color of coke and the color of coke refrigerator are very bright which contrasts sharply with the background color, and the convex dominance is high.

Fig. 3 the stewardess is in the central position, and the significance is high. The composition shows the focus of the interactive meaning and the narrative meaning. Character occupies one-half of the graph. The slogan "There's nothing more refreshing in the blue sky as delicious Coca-Cola. Ice-cold and soaring with the taste of real in-flight refreshment. Ask for the genuine goodness of Coca-Cola. Thirst asks nothing more." located at the bottom of the print advertisement which is the location of the real information. And it reflects the reliability and the authenticity of the content of the utterance. The brand identity is located at the location of the known information, Advertising quality carries on." is located in the area of unknown information. It tells us that the brand will continue to improve, so that viewers are full of expectations. At the top of the picture is the ideal message, "fly...refreshed" shows that the ideal condition for flying is to drink coke on the way.

\section{Conclusion}

In the American Coca-Cola print advertisement, the character image accounts for about $1 / 2$ of the whole advertisement picture, the expression of the characters on the screen is clearly visible, most of them are Coca-Cola in hand, their cheeks are ruddy, and they drink happily. it is an equal gesture for women to appear independently or at the same time as male images. For example, in a Coca-Cola advertisement, there are images of men and women sitting side by side in the picture, and men are pouring Coca-Cola for the women around them. We can know that the professional characteristics of characters are very obvious, including airline stewardess soldiers and so on.

In Coca-Cola advertising in the United States, the character's perspective is to look at consumers and directly show the pleasure of drinking Coca-Cola. The United States is a low-context culture that emphasizes straight- 
forward expression and directly tells consumers the benefits of their products. In the early stage of brand construction, it is necessary to cultivate and construct the consumer group's cognition of new products and new culture through advertising. The audience will only accept the loyalty to the brand until they understand the product first. Coca Cola Advertising in the United States turned to perceptual appeal in 1935, emphasizing the good feeling of drinking Coca Cola. As the advertisement says, "There' $s$ nothing more refreshing in the blue sky as delicious Coca-Cola. Ice-cold and soaring with the taste of real in-flight refreshment." The attraction of the product is not only its own edible function, but also the social function of the product. The satisfaction of socialized needs becomes more and more important. At this time, Coca-Cola is not only a kind of soft drink, but also a link of interpersonal communication, which is undoubtedly to promote the product from the perspective of sensibility rather than rationality.

\section{Reference}

1. Li zhanzi. Social semiotic analysis of multimodal discourse [J]. Foreign language studies, 2003(5):1-8.

2. Halliday, M. A. K. An Introduction to Functional Grammar (the second edition) [M]. London: Edward Arnold, 1994.

3. Halliday, M. A. K. Language as Social Semiotic: The Social Interpretation of Language and Meaning [M]. London: Edward Arnold, 1978.

4. Kress, G. \& Leeuwen, Theo Van. Reading Images: The Grammar of Visual Design [M]. London: Routledge, 2006. 